\title{
From deep learning to dark fields - medical imaging physics in ECR 2020
}

\author{
Mika Kortesniemi ${ }^{1}$ (D) \\ Received: 19 May 2020 / Accepted: 24 June 2020 / Published online: 3 July 2020 \\ (C) European Society of Radiology 2020
}

The technology in medical imaging has evolved fast and continuously on all its fronts. Many aspects related to image quality and patient safety have also improved significantly along with the technology. In general, medical imaging diagnostics and procedures have become more accurate in spatial and temporal resolution, extended in coverage, quickened in speed of exam and widened on dimensionality of information. All this has contributed to improvement of diagnostic quality and effectiveness. The latest physical and technical advancements relate to use of artificial intelligence in various aspects of medical imaging: radiation dosimetry, image reconstruction, image enhancement, image analysis and an increasing spectrum of clinical applications. Imaging hardware has also evolved to strengthen the prerequisites for better physical image quality, leading ultimately to higher sensitivity and specificity of diagnostics. The original program for the ECR included an outstanding compilation of altogether 30 sessions involving physics in medical imaging. In this editorial, the focus is in the scientific presentation including originally scheduled 52 talks. This editorial refers to the ECR 2020 Book of Abstracts [1] and the presentation code references are written in brackets in the following text. The presentations cover four main categories in the field of medical imaging: advances in CT, advances in MRI, artificial intelligence and quality in connection with safety.

\section{Advances in CT}

The advances of CT clearly represent the variability of technical fronts within radiology. Spatial resolution reaching $0.25 \mathrm{~mm}$ has been achieved with relatively unchanged noise

Mika Kortesniemi

mika.kortesniemi@hus.fi

1 HUS Medical Imaging Center, University of Helsinki and Helsinki University Hospital, POB 340, FI-00029 Helsinki, Finland and dose levels, also utilising the new reconstruction techniques (RPS 113-1, RPS 613-6). The traditional energy integrating detectors in CT are gradually followed by the first clinically applied models of photon-counting detectors where each X-ray photon energy can be measured and used for image calculation. These studies of photon-counting CT (PCCT) are demonstrating capability for whole-body ultrahigh resolution with reduced noise. Furthermore, this evolved CT scanning technique may provide context-sensitive images separately focusing on bone or soft tissue structures, improving clinical workflow and increasing information content (RPS 113-2, RPS 113-3). Metal artefact correction may be combined in the new PCCT technique to bring further improvements to CT image quality also with patients with metal implants (RPS 113-4). Image quality determination plays an essential role in the overall optimisation in CT. The studies report new developments also in this area, both by physical phantoms implemented in various forms by using $3 \mathrm{D}$ printing (RPS 113-5, RPS 113-13, RPS 113-14) and by computational methods by applying model observers to prove the benefits of scanner technology advancements (RPS 113-6). However, there are also potential pitfalls in the clinical CT scan process which should not be overlooked. One example is the patient off-centering which may cause impact on the specific CT diagnostic tasks: as iodine quantification in dual-energy CT (RPS 113-8).

\section{Advances in MRI}

Main modality-specific advances were presented also for MRI. Retrospective motion correction methods were used to create high-resolution T2SE (T2 spin echo) artefactsuppressed images in prostate imaging (RPS 1013-1). A new prototype volume-interpolated breath-hold examination (VIBE) technique used with compressed sensing showed how sparse data may be used efficiently (RPS 1013-2). As with CT (RPS 113-10, RPS 113-11, RPS 113-12), MRI 
volumetric data may be used for extraction of radiomic features and further in e.g. predictive models. As an example, MRI radiomic features were used for classification of patients between responders and non-responders to neo-adjuvant chemotherapy (NAC). Although not sensitive enough for clinical routine use, this predictive model could indicate the potential of the applied 3-T DCE-MRI-based radiomics for the care outcome prediction (RPS 1013-3). In another study, MR field non-uniformities of breast MRI were compensated on retrospective data sets as a prerequisite step to compute robust radiomic features (RPS 1013-4). The level of uncertainty is an important factor which should be understood thoroughly in any new diagnostic methods. Repeatability and reproducibility of radiomic features were investigated in MRI with a dedicated pelvis phantom showing excellent repeatability with only quarter of the radiomic features after repositioning of the pelvis phantom between scans (RPS 1013-5). Moving to contrast enhancement, from previous studies, it is known that gadolinium $(\mathrm{Gd})$ can be retained in various tissues in patients. Still, the exact mechanism of Gd deposition in the body tissue is not fully understood. Therefore, it was investigated how the biochemical process of transchelation of $\mathrm{Gd}$ ions from $\mathrm{Gd}$ based contrast agents to glycosaminoglycans (GAGs) can serve as an explanation for the long-term enhancements observed in vivo conditions. This was proved with the observed effect on T1-weighted contrast due to high relaxivities of the Gd-GAG complexes (RPS 1013-6). Novel acquisition and analysis protocols quantifying the in vivo subvoxel composition of the brain with non-parametric diffusion tensor distributions were also reported (RPS 1013-9). This addressed the problem of conventional diffusion tensor imaging (DTI) metrics in interpretation of specific tissue properties (RPS 10137). Also related to DTI, two different algorithms for fibre tract reconstruction of white matter in the brain of epilepsy patients were studied showing geometrical differences that should not be overlooked in the neurosurgery process and its accuracy (RPS 1013-8). Finally, for MRI, in vivo C13 magnetic resonance spectroscopy (MRS) has shown potential to monitor changes in lipid composition of body fat which can provide an indicator of nutritional status and other related human disorders (RPS 1013-10). Multimodal approaches were also represented. A study describing a training phantom for multiparametric ultrasound found correlations related to fatty liver disease parameters between US and MRI by using phantom data (RPS 1013-11).

\section{Artificial intelligence revising the physics in medical imaging}

Artificial intelligence (AI) is likely to change many areas of science, technology and medicine. Many papers in ECR were addressing this change by showing the versatility of $\mathrm{AI}$ in different methods and tasks within the radiology context. Most of the studied AI techniques are concentrating on a specific subset of AI, namely deep learning (DL) which belongs to a larger concept of machine learning (ML) implying an AI method which learns directly from the data. Recent DL techniques have been applied to image reconstruction and image enhancement in CT demonstrating clearly improved image quality as regards lower noise levels and well-maintained spatial resolution (RPS 613-1, RPS 613-4, RPS 613-7, RPS 6138, RPS 613-9, RPS 113-15) (see Fig. 1 for a paediatric abdominal example). More specifically, the DL image reconstruction (DLIR) may keep the image texture similar to traditional filtered-back projection (FBP)-based CT image reconstruction (RPS 613-2, RPS 613-3, RPS 613-5, RPS 613-11). This contrasts with many previously studied CT iterative reconstruction (IR) techniques where the image texture has changed compared with FBP. DL can be applied also with very sparse data as demonstrated with only 4 CBCT projections used for interventional tool reconstruction (RPS 613-12). However, DL is a new method and provides a very different approach to medical imaging. Therefore, it is also fundamentally important to perform sufficient clinical and technical validation to new AI-based methods, whether applying them in image reconstruction or further on the diagnostic tasks. Physics studies have proven the validity of DLIR methods mainly with physical phantoms. General reliability in clinical level with highly variable patient objects and clinical cases still needs more proof.

\section{Improving quality to build safety}

The fourth area of scientific presentations included qualityand safety-related topics. Relatively high radiation exposure in CT was the focus point in a European study (EUCLID project) on clinical diagnostic reference levels (DRLs). The study described clinical indication-specific use of achievable dose levels and DRLs for dose optimisation (RPS 1113-1). Another similar study concentrated on the paediatrics which is a highly relevant target population for optimisation due to the elevated radiation risk of children (RPS 1113-2). Further on the data monitoring side, radiation exposure monitoring systems have been increasingly implemented in imaging departments to enable wider scale tools for follow-up and dose optimisation. Recurrent imaging and cumulative doses of oncological patients were assessed with one of those systems aiming to improved care and safety on this patient group (RPS 1113-3). Regarding radiation dosimetry, patientspecific radiation dose estimation is a computationally demanding task when higher accuracy is needed. Computational time for interventional radiology patient dose calculation was shown to be possible in just a few seconds with GPU (graphics processing unit) accelerated dose 


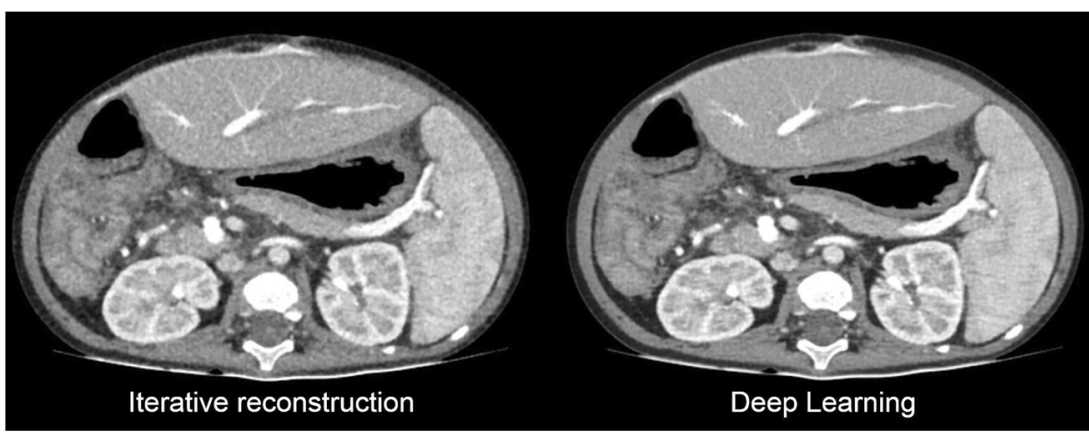

Fig. 1 Abdominal contrast-enhanced portavenous phase $2 \mathrm{~mm}$ axial CT images of a 1-year-old male patient produced by iterative reconstruction (left, ASiR-V60) and deep learning-based image reconstruction (right, DLIR-High) demonstrating the image quality difference between the

estimation (RPS 1113-5). On one of the most novel parts of radiology technology development, dark-field radiography systems are emerging into clinical studies providing a new and fascinating update of the radiography image formation. Preliminary results show that X-ray dark-field radiography can probe the lung's underlying microstructure, which has been inaccessible with traditional medical imaging modalities. In particular, the application of dark-field radiography for early detection of COPD can be considered a clear breakthrough (RPS 1113-6). In another paper, scatter artefact reduction could be developed with dark-field chest radiography, improving further the accuracy of dark-field images (RPS 1113-7). Evolving mammography technology was covered in papers representing the potential of combined imaging (digital breast tomosynthesis plus synthetic 2D mammogram or conventional full-field digital mammography) and new highresolution 3D digital breast tomographic imaging systems with $70-\mu \mathrm{m}$ voxel size (RPS 1113-10, RPS 1113-11). As a cross-over quality topic, software tools used in routine quality control tests could also be used to test the QC phantom quality and detect possible manufacturing defects in phantoms (RPS 1113-12). Finally, the evaluation of the quality of education in radiation protection courses in Germany could be used to improve harmonisation of training also across Europe.

Overall, the physics presentations for ECR 2020 provide excellent examples of the versatility and rapid development throughout the radiology modalities, computational techniques and computer algorithms. methods. Radiation dose was $\mathrm{CTDI}_{\mathrm{vol}}=1.6 \mathrm{mGy}$ and DLP $=$ $22.4 \mathrm{mGycm}$; tube voltage was $80 \mathrm{kV}$. Images courtesy of HUS Medical Imaging Center, New Children's Hospital, Helsinki, Finland

Funding information The author states that this work has not received any funding.

\section{Compliance with ethical standards}

Guarantor The scientific guarantor of this publication is Mika Kortesniemi.

Conflict of interest The author of this manuscript declares no relationships with any companies, whose products or services may be related to the subject matter of the article.

Statistics and biometry No complex statistical methods were necessary for this paper.

Informed consent Written informed consent was not required for this study because this work is an Editorial.

Ethical approval Institutional Review Board approval was not required because this work is an Editorial.

\section{Methodology}

- retrospective

- observational

\section{Reference}

1. ECR 2020 Book of abstracts (2020) Insights Imaging 11:34

Publisher's note Springer Nature remains neutral with regard to jurisdictional claims in published maps and institutional affiliations. 